Gynäkol Rundsch 1989;29:I-VI

\title{
Contents, Vol. 29, 1989
}

\section{Inhaltsverzeichnis Vol. 29,1989}

Nr. 1 Originalarbeiten

Beziehungen zwischen uteroplazentarer Hämodynamik und fetalem respiratorischem Verhalten bei Schwangerschaft mit drohender Frühgeburt oder intrauteriner Retardierung Ruckhäberle, K.-E.; Faber, R.; Petzold, J.; Forberg, J 1

Mütterliche Lipoproteine post partum. Qualitative und quantitative Untersuchungen über den Einfluss der Laktation

Brockerhoff, P.; Stinner, B.; Holzer, A.; Friese, K.; Schicketanz, K.H.; Rathgen, G.H. 12 Débimétrie fæale au moyen d'un Doppler pulse multicanaux dans Гaorte et la veine ombilicale. Résultats préliminaires dans la grossesse normale

Gasser, B.; Ditesheim, P.J.; Willemetz, J.C 22

Impedanzkardiographie in der Geburtshilfe: Die klinische Bedeutung einiger hämodynamischer Parameter

Moggian, G.; Magrone, F.; Spadazzi, A.P.; Gunelli, R.; Cicchetti, G.; Pini, P.L. ... 32

Kurzfassungen von Zeitschriftenartikeln

Amniotisches immunreaktives Trypsin in Schwangerschaften mit normalem und pathologischem Ausgang

Laroche, D.; Herlicoviez, M; Pasquet, C; Travert, G.; Fernandez, Y 35

Blasensymptomatik und Harninkontinenz nach nichtradikaler Hysterektomie

Vervest, H.A.M.; de Jonge, M.K.; Vervest, T.M.J.S.; Barents, J.W.; Haspels, A.A. . 37 Die übliche intravenöse pulsatile GnRH-Therapie führt beim Syndrom des polyzystischen

Ovars nicht zur Ovulation

Wilson, J.M.; Traub, A.I.; Sheridan, B.; Thompson, W.; Atkinson, A.B 38

Behandlung mit einem intranasalen oder subkutan verabreichten LH-RH-Agonisten zur

Unterdrückung der Ovarialfunktion bei der Behandlung der Endometriose

Lemay, A.; Maheux, R.; Huot, C; Blanchet, J.; Faure, N 40

Vergleichsstudie zwischen Panoramahysteroskopie mit gezielter Biopsie und Dilatation mit Kürettage - eine Untersuchung an 276 Fallen

Gimpelson, R.J.; Rappold, H.0 42

Magnesiumverabreichung in der Schwangerschaft. Eine Doppelblindstudie

Spätling, L.; Spätling, G 43

Erhöhte Konzentration von Prostaglandin-E-Rezeptoren bei Frauen mit Menorrhagie

Adelantado, J.M.: Rees, M.C.P.; Lopez Bernal, A.; Turnbull, A.C 45

Das psychosexuelle Trauma eines pathologischen Zervixabstrichs

Campion, M.J.; Brown, J.R.: McCance, D.J.; Atia, W.; Edwards, R.; Cuzick, J.; Singer, A. 46

Beratung von 85 schwangeren Alkoholikerinnen: Auswirkungen auf Trinkgewohnheiten

und fetale Entwicklung

Halmesmaki, E $\quad 48$

Inhaltsverzeichnis 
III

Fortbildung

Schmerzerfassung und Mehrstufenplan in der gynäkologischen Onkologie

Mitterschiffthaler, G 50

Behandlung des Tumorschmerzes

Lahousen, M 55

Kontinuierliche subkutane Opiatinfusion

Drexel, H.; Patsch, J.R 59

Mitteilung 62

Veranstaltungskalender $\quad 63$

Nr. 2 Originalarbeiten

Mammographie und Ultraschall in der Diagnose des klinisch okkulten Mammakarzinoms

Gyr, Th.; Almendral, A.C.; Meier, D 65

Der Spiegel als Partizipationsmöglichkeit bei der gynäkologischen Routineuntersuchung: Auswirkungen auf das psychische Befinden der Frau

Blaser, A.; Werren, S.; Brügger, D.; Dreher, E 69

Behandlungsresultate von 177 Carcinoma colli uteri Stadium lb und Ha an der Universi-

täts-Frauenklinik Zurich

Hammer, F.; Schreiner, W.E 78

Kurzfassungen von Zeitschriftenartikeln

Einfluss von zyklischem Adenosinmonophosphat auf die Freisetzung von humanem Choriongonadotropin und Östradiol aus kultivierten humanen Plazentazellen

Benoit, J.; Rodway, M.; Yuen, B.H.; Leung, P.C.K 93

Rauchen und zervikale intraepitheliale Neoplasie: Nikotin- und Kotininkonzentration im Serum und Zervikalschleim bei Raucherinnen und Nichtraucherinnen

Hellberg, D.; Nilsson, S.; Haley, N.J.; Hoffman, D.; Wynder, E 94

Effizienz eines fetopelvinen Index bei einem Geburtsgewicht von $4000 \mathrm{~g}$ und dariiber:

Vorläufige Ergebnisse

Morgan, M.A.; Thurnau, G.R 96

Immunszintigraphie der Endometriose des Ovars: Vorläufige Ergebnisse

Kennedy, S.H.; Soper, N.D.W.; Mojiminiyi, O.A.; Shepstone, B.J.; Barlow, D.H. ... 97

Behandlung der Endometriose durch vaginale Applikation von Gestrinone

Countinho, E.M.; Azadian-Boulanger, G 99

Klinischer Nutzen der Bestimmung der CA-125-Konzentration in Serum und Menstruationsblut

Takahashi, K.; Nagata, H.; Kijima, S.; Kusakari, M.; Shirai, T.; Yoshino, K; Kitao, M. 101

Erhöhte Kortisolwerte bei Frauen mit funktioneller hypothalamischer Amenorrhö

Suh, B.Y.; Liu, J.H.; Berga, S.L.; Quigley, M.E.; Laughlin, G.A.; Yen, S.S 102

Fortbildung

Gestationsdiabetes: Risiken und Diagnose

Weiss, P.A.M.; Hofmann, H.M.H 105

Gestationsdiabetes: Therapie und Qualitätskontrolle der Betreuung

Hofmann, H.M.H.; Weiss, P.A.M.; Urdl, W 110

Gestationsdiabetes: Screening und Therapie an der Universitätsklinik für Frauenheil-

kunde, Innsbruck

Drexel, H.; Huter, O.; Patsch, J.R 114

IV 
Inhaltsverzeichnis

Postpartales Screening nach Gestationsdiabetes

Hofmann, H.M.H.; Kettenbach, J.; Mrak, P.; Haas, J.; Rollett, H.; Weiss, P.A.M. . 118

Insulinpflichtiger Diabetes mellitus: Minimierung des perinatalen Risikos durch konsequente Euglykämie

Feiks, A.J.; Howorka, K.; Nowotny, Ch.; Waldhäusl, W. 120

Veranstaltungskalender 128

Nr. 3 Übersichtsarbeit

Polytrauma in der Schwangerschaft

Bettex, J.-D.; Schneider, H 129

Originalarbeit

Das Pena-Shokeir-Syndrom

Zeller, R.; Benz, J 148

Kurzfassungen von Zeitschriftenartikeln

Transkutan gemessene CC \&gt; 2-Spannung im Blut des Feten während der Geburt

Nickelsen, C; Weber, T 153

Schwangerschaft und Rauchen: Ergibt sich durch den Zeitpunkt der Beendigung des Rauchens ein unterschiedlicher Einfluss auf die Schwangerschaft?

MacArthur, C; Knox, E.G 154

Immunszintigraphie mit 131I-markierten HMFG2 und F(ab')2 zur präoperativen Auffindung

von klinischen und subklinischen Lymphknotenmetastasen bei Patientinnen mit Brustkrebs

Athanassiou, A.; Pectasides, D.; Pateniotis, K.; Tzimis, L.; Natsis, P.; Lafi, A.; Arapan-

toni, P.; Koutsiouba, P.; Taylor-Papadimitriou, J.; Epenetos, A 156

Zervikovaginal- und Endometriumzytologie beim Ovarialkarzinom

Takashina, T.; Ono, M.; Kanda, Y.; Sagae, S.; Hayakawa, O.; Ito, E

Epitheliale Borderlinetumoren des Ovars: intraepitheliale Neoplasie des Ovars

Yoonessi, M.; Crickard, K.; Celik, C; Yoonessi, S 159

Die Behandlung mit einem humanen Menopausengonadotropin bei Frauen mit ovulatori-

schen Zyklen und einer idiopathischen Infertilität

Welner, S.; DeCherney, A.H.; Lake Polan, M

Klinische Bestimmung des Ovarialtumor-Antigens NB/70K: Assay unter Verwendung monoklonaler Antikörper zur Unterscheidung von Ovarialkarzinomen von anderen

gynäkologischen Erkrankungen

Knauf, S 163

Ovariale Borderline-Tumore

Chambers, J.T.; Merino, M.J.; Kohorn, E.I.; Schwartz, P.E 165

Fortbildung

Immunologischer Nachweis von Infektionen mit Neisseria gonorrhoeae

Kosian, K.; Schieder, K.; Bieglmayer, C 168

Vaginale Noxen und zervikale Läsionen

Hosmann, J.; Czerwenka, K.; Schön, H 170

Die teratogene Wirkung von Infektionen der Mutter auf die Frühschwangerschaft

Arzt, W.; Tews, G.; Mursch, G.; Fröhlich, H 172

Erregerspektrum, Klinik und Laborwerte bei aszendierenden Infektionen in der Gynäkologie

Spitzer, D.; Hagen, F.; Staudach, A 175

Inhaltsverzeichnis 
$\mathrm{V}$

Immunologischer Nachweis genitaler Chlamydieninfektionen in Gynäkologie und Geburtshilfe

Schieder, K.; Kosian, K.; Bieglmayer, C 177

Chlamydieninfektion und Schwangerschaft - Routinescreening und Therapie bei 3561

Graviden

Bülau, A.; Niebauer, B.; Maly, B 180

Einmalgabe von $1 \mathrm{~g}$ Ceftriaxon versus 3 X $1 \mathrm{~g}$ Cefotaxim zur Behandlung gynäkologischer

Infektionen - eine randomisierte Vergleichsstudie

Gerstner, G.J 182

Intrauterine Übertragung der Hepatitis A: ein Fallbericht

Radivojevic, K.; Rosenkranz, M.; Weninger, M 186

Frauen und AIDS: Die Situation in Österreich und die Rolle des Gynäkologen in der Prevention

Gredler, B.; Gerstner, G.J 188

Veranstaltungskalender 192

Nr. 4 Originalarbeiten

Mamillenstimulation zur Weheninduktion beim Belastungstest

Meyer, L.; Heinzl, S 193

Das Dysurie-Syndrom. Die Bedeutung der Harnröhrenweite für die rekurrierende bakte-

rielle Zystitis der Frau

Schilling, A.; Danninger, T.; Friesen, A 200

Kurzfassungen von Zeitschriftenartikeln

Vergleich der Darstellung von Resttumorgewebe bei Ovarialkarzinompatientinnen mittels

Immunszintigraphie mit Jod-131-markierten monoklonalen Antikörpern HMFG2 und HMFG]

$\mathrm{F}(\mathrm{ab}$ ')2 gegenüber der Computertomographie

Pectasides, D.; Pateniotis, K.; Tzimis, L.; Trapalli, X.; Natsis, P.; Arapantoni, P.; Tay-

lor-Papadimitriou, J.; Epenetos, A.; Koutsiouba, P.; Athanassiou, A 215

Die Bestimmung von IgA Antikörpern gegen «Human Papillomavirus» aus dem Zervikal-

sekret von Patientinnen mit zervikaler intraepithelialer Neoplasie

Dinner, L.; Bekassy, Z.; Jonsson, N.; Moreno-Lopez, J.; Blomberg, J 217

Zur Pathogenese des Genitalprolaps und der Stressinkontinenz: Eine histologische und histochemische Studie

Gilpin, S.A.; Gosling, J.A.; Smith, A.R.B.; Warell, D.W 219

Die Bedeutung der partiellen Denervierung des Beckenbodens bei der Ätiologie des Geni

talprolaps und der Stressinkontinenz: Eine neurophysiologische Studie

Smith, A.R.B.; Hosker, G.L.; Warell, D.W 220

Die tubokorneale Anastomose und deren Prognose in Abhängigkeit von chirurgischen

Überlegungen und zusätzlichen Sterilitätsursachen

Gillett, W.R.; Herbison, GP 222

Effektivität von vaginal appliziertem Bromocriptin zur Behandlung von Patientinnen mit

Hyperprolaktinämie

Kletzky, O.A.; Vermesh, M 223

Vermessung des Plazentavolumens im zweiten Trimester: Vorhersage des «fetal outcome»

Wolf, H.; Oosting, H.; Treffers, P.E 226

Die Wirkung von Östrogen auf die Urethra bei Frauen mit Stressinkontinenz

Bhatia, N.N.; Bergman, A.; Karram, M.M 228 
VI

Inhaltsverzeichnis

Fortbildung

Therapiemöglichkeiten des Klimakteriums

Huber, J.C 230

Osteoporose und Östrogene

Metka, M 232

Kontrazeption im Klimakterium

Fischl, F 237

Neurophysiologische Vorgänge bei der Entstehung von klimakterischen Hitzewallungen

Schurz, B.; Metka, M.; Heytmanek, G.; Kurz, Ch.; Enzelsberger, H241

Die Beeinflussung von Schilddrüsen-Dysfunktionen durch Hormonsubstitution im Klimakterium

Kurz, C; Metka, M.; Enzelsberger, H.; Heytmanek, G.; Schurz, B.; Huber, J.; Spona, J. 243

Serumosteocalcin (BGP) als «Marker» für den Knochenmineralgehalt bei postmenopausa-

len Frauen

Heytmanek, G.; Metka, M.; Enzelsberger, H.; Huber, J.; Schurz, B.; Woloszczuk, W. . 245

Veranstaltungskalender

Autorenregister 249

Suppl. $1 \quad$ Festschrift für W.E. Schreiner

Suppl. 2 Jahrestagung der Bayerischen Gesellschaft für Geburtshilfe und Frauenheilkunde

und

der Österreichischen Gesellschaft für Gynäkologie und Geburtshilfe

Innsbruck, 1989

Suppl. 3 200-Jahresfeier der I. Universitäts-Frauenklinik Wien

Wien, 1989

S. Karger · Verlag für Medizin und Nalurwissenschaften

Basel · München · Paris · London · New York $n$ New Delhi · Bangkok · Singapore •Tokyo ·

Sydney

Dosierungsangaben von Medikamenten

Autoren und Verlag haben alle Anstrengungen unter-nommen, um sicherzustellen, dass Auswahl und Dosierungsangaben von Medikamenten im vorliegenden Text mit den aktuellen Vorschriften und der Praxis überein-stimmen. Trotzdem muss der Leser im Hinblick auf den Stand der Forschung, Änderungen staatlicher Gesetzge-bungen und den ununterbrochenen Fluss neuer Forschungsergebnisse bezüglich Medikamenten wirkung und Nebenwirkungen darauf aufmerksam gemacht werden, dass unbedingt bei jedem Medikament der Packungs-prospekt konsultiert werden muss, um mögliche Änderungen im Hinblick auf Indikation und Dosis nicht zu übersehen. Gleiches gilt für spezielle Warnungen und Vorsichtsmassnahmen. Ganz besonders gilt dieser Hin-weis für empfohlene neue und/oder nur selten gebrauchte Wirkstoffe.

Alle Rechte vorbehalten.

Ohne schriftliche Genehmigung des Verlags dürfen diese Publikation oder Teile daraus nicht in andere Sprachen übersetzt oder in irgendeiner Form mit mechanischen oder elektronischen Mitteln (einschliesslich Fotokopie, Tonaufnahme und Mikrokopie) reproduziert oder auf einem Datenträger oder einem Computersystem gespei-chert werden.

Copyright 1989 by

S. Karger AG, P.O. Box, CH-4009 Basel (Schweiz)

Printed in Switzerland by Thür AG Offsetdruck, Pratteln 